PROCEEDINGS OF THE

AMERICAN MATHEMATICAL SOCIETY

Volume 128, Number 10, Pages 3009-3016

S 0002-9939(00)05713-0

Article electronically published on April 28, 2000

\title{
REAL ANALYTIC STRUCTURES \\ ON A SYMPLECTIC MANIFOLD
}

\author{
FRANK KUTZSCHEBAUCH AND FRANK LOOSE
}

(Communicated by Leslie Saper)

\begin{abstract}
We prove that every symplectic manifold possesses a real analytic structure. Moreover this structure is unique up to isomorphism.
\end{abstract}

\section{INTRODUCTION}

The purpose of this note is to prove the following

Theorem. Any symplectic manifold possesses a unique (up to isomorphism) real analytic structure.

To be more precise, let $M$ be a smooth (i.e., $\mathcal{C}^{\infty}$ ) manifold and $\omega$ a smooth symplectic form on $M$. Then the existence part of the theorem states that there exists a real analytic manifold $M^{a}$ together with a real analytic symplectic form $\omega^{a}$ and a diffeomorphism $f: M^{a} \rightarrow M$ satisfying $f^{*} \omega=\omega^{a}$. On the other hand, the uniqueness part of the theorem says the following. Suppose that $\left(M_{1}, \omega_{1}\right)$ and $\left(M_{2}, \omega_{2}\right)$ are real analytic symplectic manifolds and suppose that there exists a smooth symplectomorphism $f: M_{1} \rightarrow M_{2}$, i.e., $f^{*} \omega_{2}=\omega_{1}$. Then there exists also a real analytic symplectomorphism.

The idea of the proof is essentially a combination of some well-known facts in differential topology due to $\mathrm{H}$. Whitney, in analytic geometry due to $\mathrm{H}$. Grauert and in symplectic geometry due to J. Moser. In fact, by a theorem of Whitney [15] one may assume that $M$ is already a real analytic manifold and one has to look for a smooth diffeomorphism $f$ of $M$ transforming a given smooth symplectic form $\omega$ into a real analytic symplectic form $\omega^{a}$. To this end, using Grauert's embedding theorem [1] and Whitney's approximation theorem 14, one can assume that there exists a real analytic symplectic form $\omega^{a}$ quite near to $\omega$ in the sense of a strong topology, the so-called Whitney topology. Overcoming some technical difficulties this shows then that the Moser deformation trick 11 yields in fact a diffeomorphism of $M$ transforming $\omega$ into $\omega^{a}$.

\section{Proof of the existence part}

So let $M$ be a smooth manifold and $\omega$ a smooth symplectic form on $M$. By Whitney's theorem we may assume that $M$ is real analytic and we have to find a

Received by the editors December 9, 1998.

1991 Mathematics Subject Classification. Primary 53C15; Secondary 32C05.

The first author was partially supported by SNF (Schweizerische Nationalfonds).

(C)2000 American Mathematical Society 
diffeomorphism $f$ of $M$ such that $f^{*} \omega=: \omega^{a}$ is real analytic. Of course, $f^{*} \omega$ is symplectic anyway since $d\left(f^{*} \omega\right)=f^{*}(d \omega)=0$ and $\left(f^{*} \omega\right)^{n}=f^{*}\left(\omega^{n}\right) \neq 0$ everywhere on $M$ where $2 n$ is the dimension of $M$.

Next, by Grauert's theorem, we may assume further that $M$ is embedded properly into some euclidean space $\mathbf{R}^{N}$ and using Whitney's extension theorem we may also assume that $\omega$ is defined on a fixed neighborhood $\Omega \subseteq \mathbf{R}^{N}$ of $M$.

Recall now the basic notion of the Whitney topology for smooth functions (and also for differential forms) on $\Omega$ given by the following. If $g \in \mathcal{E}(\Omega)$ where $\mathcal{E}(\Omega)$ denotes the smooth functions on $\Omega$, then the basic neighborhoods of $g$ are given by the data $K=\left(K_{j}\right)_{j \in \mathbf{N}}, m=\left(m_{j}\right)_{j \in \mathbf{N}}$ and $\varepsilon=\left(\varepsilon_{j}\right)_{j \in \mathbf{N}}$ where $\left(K_{j}\right)$ is a compact exhaustion of $\Omega, \Omega=\bigcup K_{j}, K_{j} \subseteq \operatorname{int}\left(K_{j+1}\right), m_{j} \in \mathbf{N}$ and $\varepsilon_{j}>0$. Then $h$ is in the neighborhood of $g$ corresponding to the data $(K, m, \varepsilon)$, if the difference of $h$ and $g$ on $K_{j+1} \backslash \operatorname{int}\left(K_{j}\right)$ is smaller than $\varepsilon_{j}$ up to the $m_{j}$ 'th differentiation order, i.e.,

$$
\sum_{|\alpha| \leq m_{j}} \frac{1}{\alpha !} \sup _{x \in K_{j+1} \backslash \operatorname{int} K_{j}}\left|D^{\alpha}(h-g)\right|<\varepsilon_{j}
$$

(cf., e.g., [4] or [12]). Whitney's approximation theorem [14] then states that $\mathcal{E}^{a}(\Omega) \subseteq \mathcal{E}(\Omega)\left(\mathcal{E}^{a}\right.$ denoting the real analytic functions) is dense with respect to the Whitney topology.

Finally we recall the fundamental Moser deformation trick in symplectic geometry. Suppose that $\omega_{0}$ and $\omega_{1}$ are symplectic forms on $M$ representing the same de Rham cohomology class on $M,\left[\omega_{0}\right]=\left[\omega_{1}\right] \in H_{d R}^{2}(M ; \mathbf{R})$, which are close enough so that the straight line curve $t \mapsto \omega_{t}:=(1-t) \omega_{0}+t \omega_{1}$ has non-degenerate values in $\mathcal{E}^{(2)}(M)\left(\mathcal{E}^{(k)}(M)\right.$ denoting the differential $k$-forms on $\left.M\right)$ for all $t \in[0,1]$. Then for a choice of a potential $\beta \in \mathcal{E}^{(1)}(M)$, i.e., $-d \beta=\sigma:=\omega_{1}-\omega_{0}$, one finds a unique (smooth) curve of vector fields $t \mapsto \xi_{t}$ such that

$$
i_{\xi_{t}} \omega_{t}=\beta,
$$

thanks to the fact that $\omega_{t}$ is non-degenerate. (Here $i_{\xi} \omega$ denotes the contraction of the form $\omega$ in the first argument by the vector field $\xi$.) Taking derivatives one finds that $d i_{\xi_{t}} \omega_{t}+\sigma=0$. Finally suppose that the non-autonomous vector field $\xi_{t}$ on $M$ is integrable up to time one, i.e., there exists a curve $t \mapsto f_{t}$ in the diffeomorphism group of $M$ with $f_{0}=$ id and

$$
\frac{d}{d t} f_{t}=\xi_{t} \circ f_{t} .
$$

Then, since the Lie derivative $\mathcal{L}_{\xi}$ satisfies $\mathcal{L}_{\xi}=d i_{\xi}+i_{\xi} d$ and the forms $\omega_{t}$ are closed, one concludes that

$$
\frac{d}{d t}\left(f_{t}^{*} \omega_{t}\right)=f_{t}^{*}\left(\mathcal{L}_{\xi_{t}} \omega_{t}+\frac{d}{d t} \omega_{t}\right)=f_{t}^{*}\left(d i_{\xi} \omega_{t}+\sigma\right)=0,
$$

for all $t \in[0,1]$. This shows that $f:=f_{1}$ fulfills the requirement $f^{*} \omega_{1}=\omega_{0}$ since

$$
f^{*} \omega_{1}-\omega_{0}=f_{1}^{*} \omega_{1}-f_{0}^{*} \omega_{0}=\int_{0}^{1} \frac{d}{d t}\left(f_{t}^{*} \omega_{t}\right) d t=0 .
$$

Therefore, to prove the theorem, it is enough to find a real analytic symplectic form $\omega^{a}$ on $M$ which satisfies

(a) $[\omega]=\left[\omega^{a}\right] \in H_{d R}^{2}(M ; \mathbf{R})$;

(b) $\omega$ and $\omega^{a}$ are close enough such that $\omega_{t}:=(1-t) \omega+t \omega^{a}$ is non-degenerate for all $t \in[0,1]$; 
(c) there exists $\beta \in \mathcal{E}^{(1)}(M)$ with $d \beta=\omega-\omega^{a}$ such that the induced vector field $\xi_{t}$ is integrable up to time one.

Lemma 1. Let $M$ be a real analytic manifold and $\omega \in \mathcal{E}^{(k)}(M)$ a smooth and closed differential $k$-form on $M(k \geq 0)$. Then there exists a real analytic closed differential $k$-form $\omega^{a}$ on $M$ representing the same de Rham cohomology class, $\left[\omega^{a}\right]=[\omega] \in H_{d R}^{k}(M ; \mathbf{R})$.

Proof of Lemma 1. By a theorem of Whitney-Bruhat [16] there exists a totally real embedding of $M$ into a complex manifold $X$, say $\iota: M \hookrightarrow X$, meaning that for all $m \in M$ we have $T X_{\iota(m)}=\iota_{*} T M_{m} \oplus J \iota_{*} T M_{m}, J$ denoting the complex structure of $X$ in $\iota(m)$. Moreover, by Grauert's solution of the Levi problem [1], we may further assume that $X$ is a Stein manifold, i.e., $X$ is holomorphically separable and holomorphically convex. We may in addition assume that $\iota: M \hookrightarrow X$ is the homotopy inverse of a strong deformation retract of $X$ onto $M, \pi: X \rightarrow$ $M$, inducing therefore an isomorphism on the level of de Rham cohomology (with coefficients in $\mathbf{C}$ ). (This result is stated in 2, chap. V, $\S 4$. For more general results see also [10] and [8].) Let $\iota^{*}: H_{d R}^{2}(X ; \mathbf{C}) \rightarrow H_{d R}^{2}(M ; \mathbf{C})$ denote this induced isomorphism.

Now $H_{d R}^{2}(M ; \mathbf{C})=H_{d R}^{2}(M ; \mathbf{R}) \otimes \mathbf{C}$ and we regard $\omega$ as an element in $\mathcal{E}^{(2)}(M ; \mathbf{C})$. We want to consider now $\pi^{*} \omega \in \mathcal{E}^{(2)}(X)$. Of course, $\pi^{*} \omega$ represents a de Rham cohomology class on $X$ as well. Now, Cartan-Serre's so-called Theorem B for Stein manifolds implies that the de Rham cohomology on $X$ is representable by holomorphic forms (see [2], chap. V, $\S 4$ ). Therefore we can choose a holomorphic and closed 2-form $\omega^{\text {hol }}$ on $X$ such that $\left[\omega^{\text {hol }}\right]=\left[\pi^{*} \omega\right] \in H_{d R}^{2}(X ; \mathbf{C})$. Then

$$
\omega^{a}:=\operatorname{Re}\left(\iota^{*} \omega^{\text {hol }}\right) \in \mathcal{E}^{(2)}(M)
$$

is real analytic and represents the same cohomlogy class as the given $\omega$ proving the lemma.

Therefore we have fulfilled condition (a). However, $\omega^{a}$ is not necessarily close enough to $\omega$ to satisfy conditions (b) and (c). In fact, to fulfill condition (b) it would be sufficient to find a real analytic representative $\omega^{a}$ of $[\omega] \in H_{d R}^{2}(M ; \mathbf{R})$ in every Whitney neighborhood of $\omega$ (as an element in $\mathcal{E}^{(2)}(M)$ ). However, for condition (c) it is better that not only is $\omega$ "Whitney near" to $\omega^{a}, \omega \approx \omega^{a}$, but moreover we must find a potential $\beta \in \mathcal{E}^{(1)}(M)$, i.e., $d \beta=\omega-\omega^{a}$, which is in addition Whitney near to zero, $\beta \approx 0$. In fact, this would also imply that the induced vector field $\xi_{t}$ is Whitney near to zero, $\xi_{t} \approx 0$; in particular it goes to zero, if $x \in M$ goes to infinity, $\xi_{t}(x) \rightarrow 0$ for $|x| \rightarrow \infty$ (recall that we have embedded $M$ properly in some $\mathbf{R}^{N}$ ). In fact, by a standard argument for the range of the short time existence of the solution curve of a dynamical system $\dot{x}=\xi_{t}(x)$ on $M$, one can conclude then that $t \mapsto f_{t}(x)$ exists for all $0 \leq t \leq 1$ and all $x \in M$. So it is enough to prove the following

Lemma 2. Let $M$ be a real analytic manifold and let $\omega$ be a smooth and closed differential $k$-form on $M$. Let $B \subseteq \mathcal{E}^{(k-1)}(M)$ be an arbitrary small Whitney neighborhood of the zero $(k-1)$-form on $M$. Then there exists an element $\beta \in B$ such that $\omega^{a}:=\omega+d \beta$ is real analytic.

Proof of Lemma 2. Choose first a real analytic representative $\omega_{1}^{a}$ of $[\omega]$ on $M$ according to Lemma 1. Thus there exists an $\alpha \in \mathcal{E}^{(k-1)}(M)$ such that $d \alpha=\omega-\omega_{1}^{a}$. 
Now, by the Whitney approximation theorem, there exists a $\beta \in B \subseteq \mathcal{E}^{(k-1)}(M)$ such that $\alpha^{a}:=\alpha+\beta$ is real analytic and therefore $d \alpha^{a}$ as well. Therefore

$$
\omega+d \beta=\left(d \alpha+\omega_{1}^{a}\right)+d\left(\alpha^{a}-\alpha\right)=\omega_{1}^{a}+d \alpha^{a}
$$

is real analytic.

Altogether we have now found a real analytic symplectic form $\omega^{a}$ on $M$ satisfying conditions (a), (b) and (c) and we have proved the existence part of our theorem.

\section{Proof of the uniqueness part}

So let $M_{1}$ and $M_{2}$ be two real analytic manifolds with real analytic symplectic forms $\omega_{1}$ and $\omega_{2}$ respectively, and let $f: M_{1} \rightarrow M_{2}$ be a smooth diffeomorphism with $f^{*} \omega_{2}=\omega_{1}$. Now, on the one hand, the homotopy class $\left\{g \in \operatorname{Diff}\left(M_{1}, M_{2}\right) \mid g \sim f\right\}$ of $f$ in $\operatorname{Diff}\left(M_{1}, M_{2}\right)$ is open, and on the other hand, using Grauert's embedding theorem, the real analytic diffeomorphisms $\operatorname{Diff}^{a}\left(M_{1}, M_{2}\right)$ are dense in $\operatorname{Diff}\left(M_{1}, M_{2}\right)$ (which proves that the real analytic structure of a smooth manifold is unique up to isomorphism, by the way). Thus there exists a real analytic diffeomorphism $f^{a} \in \operatorname{Diff}^{a}\left(M_{1}, M_{2}\right)$, arbitrarily Whitney near to $f$, and in the same homotopy class, of course. Therefore for $\tilde{\omega}_{1}:=\left(f^{a}\right)^{*}\left(\omega_{2}\right)$ we conclude that $\tilde{\omega}_{1}$ is real analytic, $\left[\omega_{1}\right]=\left[\tilde{\omega}_{1}\right] \in H_{d R}^{2}\left(M_{1}\right)$, and $\tilde{\omega}_{1}$ is Whitney near to $\omega_{1}$. Therefore it suffices to find a real analytic potential $\beta \in \mathcal{E}^{(1)}(M)$, i.e., $d \beta=\omega_{2}-\omega_{1}$, which is Whitney near to zero, $\beta \approx 0$. In fact, then Moser's deformation trick would produce a real analytic diffeomorphism $g: M_{1} \rightarrow M_{1}$ with $g^{*} \tilde{\omega}_{1}=\omega_{1}$ and therefore the diffeomorphism $f^{a} \circ g \in \operatorname{Diff}^{a}\left(M_{1}, M_{2}\right)$ is the desired real analytic symplectomorphism between $M_{1}$ and $M_{2}$.

So what we may assume now is the following. Let $M$ be a real analytic manifold and let $\omega_{0}, \omega_{1}$ be real analytic symplectic forms on $M$ such that $\left[\omega_{0}\right]=\left[\omega_{1}\right]$ and $\omega_{0}$ and $\omega_{1}$ are arbitrarily Whitney near to each other. We may also assume that there exists a smooth curve $[0,1] \rightarrow \operatorname{Diff}(M), t \mapsto f_{t}$ with $f_{0}=$ id and $f_{1}^{*}\left(\omega_{1}\right)=\omega_{0}$ which is entirely contained in an arbitrary small Whitney neighborhood of the identity. However, $f_{t}$ is only smooth and we want to find a real analytic diffeomorphism $g$ such that $g^{*}\left(\omega_{1}\right)=\omega_{0}$.

Lemma 1. (a) ("Real analytic Poincaré Lemma") There exists a real analytic $\beta^{a} \in \mathcal{E}^{(1)}(M)$ such that $d \beta^{a}=\omega_{0}-\omega_{1}$.

(b) ("Whitney-Poincaré Lemma") There exists a smooth $\beta \in \mathcal{E}^{(1)}(M)$ such that $\beta$ is Whitney near to zero, $\beta \approx 0$, and $d \beta=\omega_{0}-\omega_{1}$.

Proof. (a) We consider again a Stein tube $\iota: M \hookrightarrow X$ for $M$ and denote by $\pi: X \rightarrow$ $M$ the retraction. We look at $X$ as a germ around $M$, i.e., all neighborhoods of $\iota(M) \subseteq X$ which we use in the sequel are again denoted by $X$.

Now, since $\omega_{0}-\omega_{1}$ is real analytic, there exists a holomorphic 2-form $\omega^{\text {hol }}$ on $X$, $\omega^{\text {hol }} \in \Omega^{(2)}(X)$, such that $\iota^{*}\left(\omega^{\text {hol }}\right)=\omega_{0}-\omega_{1}$. Moreover $\iota^{*}\left(d \omega^{\text {hol }}\right)=d\left(\iota^{*} \omega^{\text {hol }}\right)=$ $d \omega_{0}-d \omega_{1}=0$ and by the identity theorem in several complex variables we conclude that $d \omega^{\text {hol }}=0$. Furthermore $\iota$ induces an isomorphism on the level of cohomology. But $\iota^{*}\left(\left[\omega^{\mathrm{hol}}\right]\right)=\left[\iota^{*} \omega^{\mathrm{hol}}\right]=\left[\omega_{0}-\omega_{1}\right]=0$ and therefore $\left[\omega^{\mathrm{hol}}\right]=0 \in H_{d R}^{2}(X ; \mathbf{C})$. Since $X$ is Stein, we have

$$
H_{d R}^{2}(X ; \mathbf{C})=\frac{\operatorname{ker}\left\{d: \Omega^{(2)}(X) \rightarrow \Omega^{(3)}(X)\right\}}{\operatorname{im}\left\{d: \Omega^{(1)}(X) \rightarrow \Omega^{(2)}(X)\right\}},
$$


where $\Omega^{(k)}(X)$ denotes the holomorphic $k$-forms on $X$. So we can choose a $\beta^{\text {hol }} \in$ $\Omega^{(1)}(X)$ such that $d \beta^{\text {hol }}=\omega^{\text {hol }}$. Now set $\beta^{a}:=\operatorname{Re}\left(\iota^{*} \beta^{\text {hol }}\right)$. Then $\beta^{a}$ is real analytic and

$$
d \beta^{a}=\operatorname{Re}\left(d \iota^{*} \beta^{\text {hol }}\right)=\operatorname{Re}\left(\iota^{*} \omega^{\text {hol }}\right)=\operatorname{Re}\left(\omega_{0}-\omega_{1}\right)=\omega_{0}-\omega_{1} .
$$

(b) Define the curve of smooth vector fields $t \mapsto \xi_{t}$ on $M$ by

$$
\xi_{t} \circ f_{t}:=\frac{d}{d t} f_{t}
$$

and set $\beta_{t}:=i_{\xi_{t}} \omega_{0}$ and finally $\beta:=-\int_{0}^{1} \beta_{t} d t$. Since $f_{t} \approx$ id for all $t \in[0,1]$, we conclude that $\xi_{t} \approx 0$ and therefore $\beta_{t} \approx 0$ and finally $\beta \approx 0$. On the other hand, we see that

$$
\begin{aligned}
\omega_{1}-\omega_{0} & =f_{1}^{*}\left(\omega_{0}\right)-f_{0}^{*}\left(\omega_{0}\right)=\int_{0}^{1} \frac{d}{d t} f_{t}^{*}\left(\omega_{0}\right) d t \\
& =\int_{0}^{1} \mathcal{L}_{\xi_{t}} \omega_{0} d t=\int_{0}^{1} d i_{\xi_{t}} \omega_{0} d t=\int_{0}^{1} d \beta_{t} d t=d\left(\int_{0}^{1} \beta_{t} d t\right)=-d \beta .
\end{aligned}
$$

Remarks. 1. Part (a) of Lemma 1 is not used in the subsequent proof. However, we think it is interesting on its own, since together with Lemma 1 of section 2 it shows that the inclusion of real analytic $k$-forms on $M$ into the smooth $k$-forms on $M$ induces an isomorphism between the de Rham cohomology groups with respect to real analytic $k$-forms and smooth $k$-forms.

2. We leave it to the reader to formulate a more quantitative version of part (b) of the lemma, i.e., how near one can choose $\beta$ to 0 in terms of how near $\omega_{0}$ is to $\omega_{1}$. The only thing we need in the sequel is that we have $\beta$ close enough to zero such that the vector fields $\xi_{t}$ given by $i_{\xi_{t}} \omega_{0}=d \beta$ are going to zero when $x \in M$ goes to infinity since we want $\dot{x}=\xi_{t}(x)$ to be integrable up to time one. Thus $\beta-$ or even better $d \beta$ - has to be closer to zero than $\omega_{0}$ is. But we can regard $\omega_{0}$ as fixed and $\omega_{1}$ we can assume as near to $\omega_{0}$ as we want which shows then that the $\beta$ constructed in part (b) satisfies the requirement.

However, the $\beta$ constructed in part (b) is not quite the correct one since it is not real analytic. What we need is a potential for $\omega_{0}-\omega_{1}$ satisfying (a) and (b) ("real analytic Whitney-Poincaré Lemma"). To this end assume that the following lemma is true.

Lemma 2. Let $\beta \in \mathcal{E}^{(1)}(M)$ be such that $d \beta$ is real analytic. Then there exists a $\gamma \in \mathcal{E}(M)$ so that $\beta+d \gamma$ is real analytic.

End of the proof of the uniqueness part of the theorem. So let $\beta$ be smooth and Whitney near to zero and $d \beta=\omega_{0}-\omega_{1}$. Choose a smooth function $\gamma$ on $M$ according to Lemma 2 so that $\beta+d \gamma=: \tilde{\beta}^{a}$ is real analytic. Next use the Whitney approximation theorem to find a smooth function $h$ on $M$ with $h \approx 0$ such that $\gamma^{a}:=\gamma+h$ is real analytic and set $\beta^{a}:=\beta-d h$. Then we have $\beta^{a} \approx 0$ and moreover $d \beta^{a}=d \beta=\omega_{0}-\omega_{1}$ and finally

$$
\beta^{a}=\left(\tilde{\beta}^{a}-d \gamma\right)-d\left(\gamma^{a}-\gamma\right)=\tilde{\beta}^{a}-d \gamma^{a}
$$

is real analytic.

We use this $\beta^{a}$ in the Moser deformation trick to find a real analytic $g \in \operatorname{Diff}(M)$ with $g^{*} \omega_{1}=\omega_{0}$ thus proving the theorem. 
For the proof of Lemma 2 we need the following ingredients. Consider again a Stein tube $\iota: M \hookrightarrow X$ with deformation retraction $\pi: X \rightarrow M$. Then it is wellknown (see e.g. [3]) that there exists a homotopy operator $I: \mathcal{E}^{(k+1)}(X) \rightarrow \mathcal{E}^{(k)}(X)$ satisfying

(i) $\omega-(\iota \circ \pi)^{*} \omega=(d I+I d)(\omega)$,

(ii) $\iota^{*} I(\omega)=0$,

for all differential forms $\omega$ on $X$. In particular one gets a relative version of the Poincaré Lemma in the sense that a closed form $\omega$ on $X$ satisfying $\iota^{*} \omega=0$ has a potential $\alpha$ satisfying $\iota^{*} \alpha=0$, namely $\alpha=I \omega$.

The other ingredient is again a consequence of Theorem B for Stein manifolds (see 2], chap. V, §4): If $X$ is Stein and $\tau \in \mathcal{E}^{(1)}(X)$ is smooth such that $d \tau$ is holomorphic, then there exists an $f \in \mathcal{E}(X)$ such that $\tau-d f$ is holomorphic.

Proof of Lemma 2. Let $\omega=d \beta$ and choose a Stein tube $\iota: M \hookrightarrow X$ with a holomorphic 2-form $\sigma$ on $X$ with $\iota^{*}(\sigma)=\omega$. By the identity principle we have $d \sigma=0$. Therefore there exists an $\alpha \in \mathcal{E}^{(1)}(X)$ such that $\iota^{*} \alpha=0$ and

$$
d \alpha=(\iota \circ \pi)^{*}(\sigma)-\sigma
$$

(namely $\alpha=-I \sigma)$. From this we see that

$$
d\left(\pi^{*} \beta-\alpha\right)=\pi^{*} \omega-d \alpha=\pi^{*} \iota^{*}(\sigma)-d \alpha=\sigma
$$

is holomorphic. Thus we can find an $f \in \mathcal{E}(X)$ such that $\pi^{*} \beta-\alpha-d f \in \Omega^{(1)}(X)$. Finally we set $\gamma:=\operatorname{Re}\left(\iota^{*} f\right)$ and conclude that

$$
\beta+d \gamma=\operatorname{Re}\left((\pi \circ \iota)^{*}(\beta)-\iota^{*}(\alpha)-\iota^{*}(d f)\right)=\operatorname{Re}\left(\iota^{*}\left(\pi^{*} \beta-\alpha-d f\right)\right)
$$

is real analytic.

\section{Concluding Remarks}

1. The following equivariant version of the theorem is also true. Let $K$ be a compact Lie group (with its natural real analytic structure). Then:

Theorem. Any symplectic $K$-manifold has a unique real analytic $K$-structure.

More precisely: If $M$ is a smooth $K$-manifold and if $\omega$ is a $K$-invariant symplectic form on $M$, then there exists a real analytic manifold $M^{a}$, on which $K$ acts real analytically, together with an invariant real analytic form $\omega^{a}$ and an equivariant symplectomorphism $f: M^{a} \rightarrow M$. Moreover, if $\left(M_{1}, \omega_{1}\right)$ and $\left(M_{2}, \omega_{2}\right)$ are real analytic $K$-manifolds and if $f: M_{1} \rightarrow M_{2}$ is a smooth equivariant diffeomorphism, then there exists also a real analytic equivariant diffeomorphism.

For the proof of the existence part we would like to mention that Whitney's theorem is true also in the equivariant case (see [5], 99]). So we may assume that $M$ is already real analytic and $K$ acts on $M$ in an analytic fashion. Now, using the average method over the compact group $K$, Lemma 1 is true also in the equivariant case, i.e., every cohomology class on $M$ can be even represented by a $K$-invariant real analytic differential form.

One difficulty arises from the fact that $M$ is in general not equivariantly embeddable in some euclidean space $\mathbf{R}^{N}$, where $K$ acts linearly. (For this one needs that 
$M$ has only finitely many orbit types.) However, the first author has proved [7] that $\mathcal{E}_{K}^{a}(M) \subseteq \mathcal{E}_{K}(M)$ is nevertheless dense with respect to the Whitney topology (which can be defined without an embedding of $M$, of course) and the same statement is true for differential forms. Therefore one finds in fact an invariant 1-form on $M$, Whitney near to zero (such that the corresponding equivariant vector field $\xi_{t}$ is integrable up to time one), so that $\omega^{a}:=\omega+d \beta$ is real analytic. Therefore Moser's method yields indeed an equivariant diffeomorphism $f$ with $f^{*} \omega=\omega^{a}$.

Similar reasoning, which we leave to the reader, shows also the uniqueness part of the theorem using essentially the averaging over the compact group.

A more general version of the theorem, namely with respect to a proper action of an arbitrary Lie group, should be true. The existence of the real analytic structure is known in that case (see 5 ). The uniqueness is not known in general. However, it has been proved in the case where the Lie group has finitely many components (see [7]) or, more generally, can be embedded into a Lie group with finitely many components (see [6]).

2. We would like to mention that when $M$ is compact, the proof of the theorem is much easier. For instance, a real analytic representative in each cohomology class can be found by taking the unique harmonic representative with respect to a real analytic Riemannian metric using Hodge's theorem and avoiding Stein theory completely. Moreover, one has no difficulties with the short time range of the flow with respect to a vector field, thus avoiding the discussions on the Whitney topology.

3. Another method in the compact case is to use the heat flow with respect to a real analytic metric (the heat flow is clearly used in the proof of the Whitney approximation theorem, too). It is well-known (and easy to see) that the heat flow for differential forms respects the closed forms and moreover the cohomology coset classes inside the closed forms. On the other hand, the heat flow is regularizing, i.e., if $t \mapsto \omega_{t}$ is the solution of

$$
\frac{d}{d t} \omega_{t}=\triangle \omega_{t}
$$

and the initial value $\omega_{0}$ is smooth, then $\omega_{t}$ is (immediately) real analytic for $t>0$. Even Moser's method can be used directly with the curve $t \mapsto \omega_{t}$ given by the heat flow instead of the straight line segment.

In the non-compact case there also exists a heat kernel on the manifold (cf. [13]). However, this gives a solution of the heat flow only for initial values for which one knows some restrictions on the asymptotics, and not in the general case. Nevertheless, we could also use this method for proving the real analytic Whitney-Poincaré Lemma in section 3 (cf. Lemma 2).

\section{ACKNOWLEDGEMENTS}

1. We would like to thank F. Knop who was the first to formulate the theorem (as far as we know at least).

2. Most of this work has been done during the stay of the first named author in Tübingen in spring 1998. We would like to thank G. Huisken for support and hospitality and for useful discussions during that time.

3. We would like to thank Erik Sommer and the referee of this paper for useful remarks and stimulating suggestions improving the first version of the paper. 


\section{REFERENCES}

1. Grauert, H., On Levi's problem and the imbedding of real-analytic manifolds. Ann. of Math. 68 (1958), 460-472 MR 20:5299

2. Grauert, H. and R. Remmert, Theory of Stein spaces. Springer-Verlag, Berlin Heidelberg New York 1979 MR 82d:32001

3. Guillemin, V. and S. Sternberg, Symplectic techniques in physics. Cambridge University Press, Cambridge 1990 MR 91d:58073

4. Hirsch, M., Differential topology. Springer-Verlag, Berlin Heidelberg New York 1988 MR 96c:57001

5. Illman, S., Every proper smooth action of a Lie group is equivalent to a real analytic action, a contribution to Hilbert's fifth problem. Ann. of Math. Stud. 138 (1995), 189-220 MR 97a:57037

6. Illman, S. and S. Kankaarinta, Some basic results for real analytic proper G-manifolds. Preprint Helsinki 1998

7. Kutzschebauch, F., On the uniqueness of the analyticity of a proper G-action. Manuscripta Math. 90 (1996), 17-22 MR 97k:57047

8. — - Eigentliche Wirkungen von Lie-Gruppen auf reell-analytischen Mannigfaltigkeiten. Schriftenreihe des Graduiertenkollegs Geometrie und Mathematische Physik, RuhrUniversität Bochum, Heft 5 (1994)

9. Matumoto, T. and M. Shiota, Unique triangulation of the orbit space of a differentiable transformation group and its applications, homotopy theory and related topics. Adv. Stud. Pure Math. 9 (1986), 41-55 MR 88g:57041

10. Mihalache, N., Special neighborhoods of subsets in complex spaces. Math. Z. 221 (1996), 49-58 MR 96j:32006

11. Moser, J., On the volume element of a manifold. Trans. Am. Math. Soc. 120 (1965), 286-294 MR 32:409

12. Narasimhan, R., Analysis on real and complex manifolds. North-Holland Publishing Company, Amsterdam 1973 MR 49:11576

13. Schoen, R. and S.T. Yau, Lectures on differential geometry. International Press, Boston 1994 MR 97d:53001

14. Whitney, H., Analytic extensions of differentiable functions defined in closed sets. Trans. Am. Math. Soc. 30 (1934), 63-89 CMP 95:18

15. — Differentiable manifolds. Ann. of Math. 37 (1936), 645-680

16. Whitney, H. and F. Bruhat, Quelques proprietés fondamentales des ensembles analytiques. Comment. Math. Helv. 33 (1959), 132-160 MR 21:889

Mathematisches Institut der Universität, Rheinsprung 21, CH - 4051 Basel, SwitzerLAND

Current address: Matematiska Institutionen, Box 480, S-751 06 Uppsala, Sweden

E-mail address: kutzsche@math.uu.se

Mathematisches Institut der Universität, Auf der Morgenstelle 10, D - 72076 TüBingen, Germany

E-mail address: frank.loose@uni-tuebingen.de 